\title{
High throughput characterization of the optical properties of compositionally graded combinatorial films
}

\author{
Peter K. Schenck ${ }^{*}$, Debra L. Kaiser, Albert V. Davydov \\ Materials Science and Engineering Laboratory, National Institute of Standards and \\ Technology, Gaithersburg, MD 20899, USA
}

\begin{abstract}
Compositionally graded combinatorial films have been characterized by a high throughput automated spectroscopic reflectometer. The data from this instrument were used to map the thickness and index of refraction of the compositionally varying films. Combinatorial films produced by dual-beam, dual-target pulsed laser deposition and characterized with the reflectometer include the $\mathrm{BaTiO}_{3}-\mathrm{SrTiO}_{3}$ system on silicon (dielectric and ferroelectric films). In addition, combinatorial Au/Ni electrical contacts on $n$-GaN/sapphire produced by electron-beam (e-beam) vaporization have been characterized with the spectroscopic reflectometer. The $\mathrm{Au} / \mathrm{Ni} / n-\mathrm{GaN} /$ sapphire structures were characterized both as-deposited and after annealing at $400{ }^{\circ} \mathrm{C}$ for $60 \mathrm{~s}$ in flowing argon.
\end{abstract}

(C) 2003 Published by Elsevier B.V.

PACS: 78.40; 07.60.H; 78.66

Keywords: Reflectometry; Thin films; Optical properties; Combinatorial

\section{Introduction}

The use of combinatorial chemistry techniques for the discovery of new materials with novel properties for use in thin film devices $[1,2]$ requires the development of rapid throughput measurement techniques to characterize the library films. This paper describes the high throughput characterization of the optical properties and thickness of combinatorial library films using a custom spectroscopic reflectometry system. This tool was first used for rapid, nondestructive thickness assays of $\mathrm{BaTiO}_{3}-\mathrm{SrTiO}_{3}$ films

\footnotetext{
${ }^{*}$ Corresponding author.

E-mail address: peter.schenck@nist.gov (P.K. Schenck).
}

of interest for electronic devices [3,4]. More recently, the tool was used for high throughput optical measurements of a $\mathrm{Au} / \mathrm{Ni}$ combinatorial library film on $n$-GaN/sapphire. GaN is of interest for optoelectronic devices such as light emitting diodes and laser diodes [5], and the $\mathrm{Au} / \mathrm{Ni}(\mathrm{O})$ system is a candidate transparent electrode $[6,7]$ material for nitride-based devices.

\section{Experimental}

\subsection{Thin film production}

The $\mathrm{BaTiO}_{3}-\mathrm{SrTiO}_{3}$ compositionally varying combinatorial library films were produced using a 
dual-beam, dual-target pulsed laser deposition (PLD) system. Pulsed laser deposition is a good prototyping tool for the study of inorganic thin films [8]. The details of the deposition system are described elsewhere [9]. The $\mathrm{Au} / \mathrm{Ni}$ on $n-\mathrm{GaN} / \mathrm{sap}-$ phire library film was produced by e-beam vaporization and is described in a separate paper in this issue [10].

\subsection{Spectroscopic reflectometry}

The spectroscopic reflectometry [11] apparatus was assembled mainly from commercially available components. The basic instrument consists of a $400 \mu \mathrm{m}$ (diameter) bifurcated fiber-optic probe illustrated in Fig. 1. Light from either a quartz halogen source, with an effective useful range of $360 \mathrm{~nm}$ to $2 \mu \mathrm{m}$, or a deuterium lamp, with an effective useful range of 200$400 \mathrm{~nm}$, is brought to the surface via one leg of the bifurcated fiber. The other leg of the bifurcated fiber collects the reflected light and couples the light to a spectrometer. Two spectrometers are used to cover the wavelength range of 180-1000 $\mathrm{nm}$. In practice the UV source is cut off at $250 \mathrm{~nm}$ to prevent solarization of the fiber-optic probe. Using a $400 \mu \mathrm{m}$ bifurcated fiber the spatial resolution of the probe was determined to be $\approx 0.5 \mathrm{~mm}$. The film/substrate specimen is mounted on an XY stage for mapping the reflectivity of the film. In practice the reflection spectra are normalized against the reflection spectra from a sample of the bare substrate. This removes the spectral variation

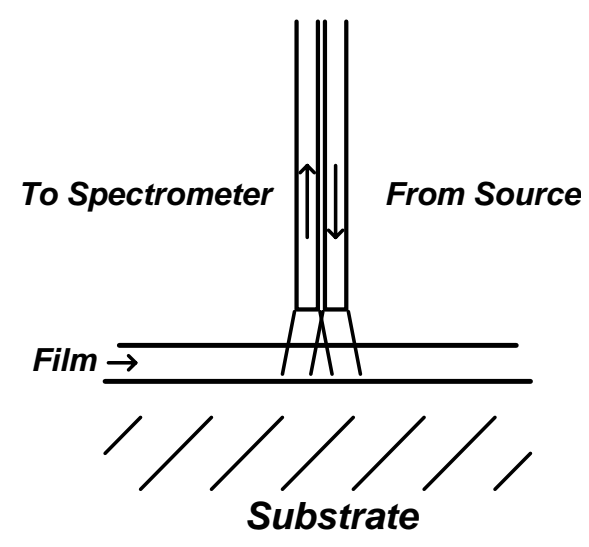

Fig. 1. Bifurcated fiber-optic probe used for spectroscopic reflectometry. of both the light source and the spectrometer, but produces a reflection spectrum relative to the substrate. The absolute reflection spectrum is obtained by multiplying the relative spectrum by a calculated spectrum for the substrate. This correction can be eliminated using a reflection standard. Mapping the reflectivity on a $1 \mathrm{~mm}$ grid pattern takes $1-2 \mathrm{~s}$ per point. The spectra are stored for later data analysis to obtain complex index of refraction information and thickness. The analysis algorithm of the spectra varies by film studied and is discussed below for each respective case. The reflection apparatus can also be used to measure transmission of semi-transparent films. This is accomplished by replacing the bifurcated fiber-optic probe with two separate fibers aligned to each other. The film/substrate specimen is then supported by its edges between the two fibers. One fiber illuminates the film and the other fiber collects the transmitted light. No reference substrate is required and the absolute transmission of the film is obtained.

\subsection{X-ray diffraction studies}

The crystal quality of $\mathrm{Au} / \mathrm{Ni}$ bi-layers on $\mathrm{GaN}$ was assessed by X-ray diffraction (XRD). $\Theta-2 \Theta$ scans were collected across the combinatorial array at ambient temperature on a scanning X-ray microdiffractometer equipped with general area detector using $\mathrm{Cu}$ $\mathrm{K} \alpha$ radiation.

\section{Results and discussion}

\subsection{Characterization of the thickness of $\mathrm{BaTiO}_{3}-\mathrm{SrTiO}_{3}$ combinatorial films}

A sample relative reflection spectrum from a $\mathrm{BaTiO}_{3}-\mathrm{SrTiO}_{3}$ film on silicon (at room temperature) is shown in Fig. 2, along with the resulting fit. The oscillations in the reflectance are caused by thin film interference between the light reflected from the two surfaces of the film. The periodicity of the oscillations is proportional to the product of the index of refraction (real part) and the film thickness. The depth of the oscillations is determined by the respective complex indices of the film and the substrate material. The spectra are further complicated by the 


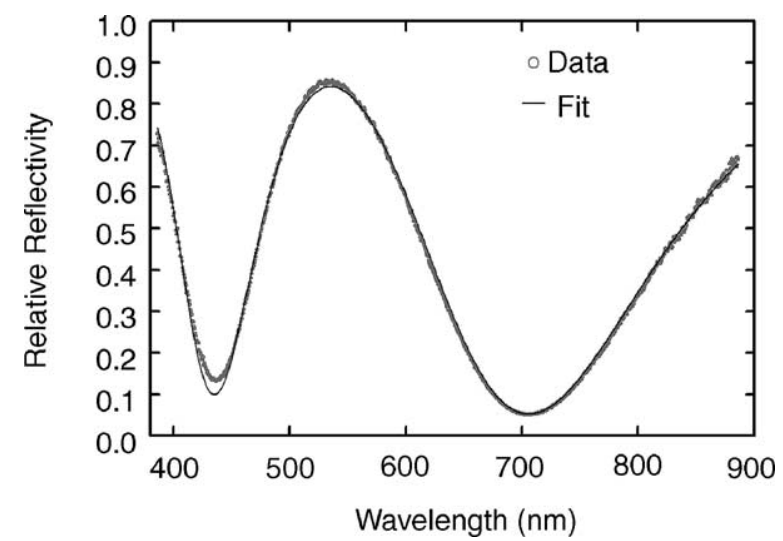

Fig. 2. Reflection spectrum from a $317 \mathrm{~nm}$ thick $\mathrm{Ba}_{x} \mathrm{Sr}_{1-x} \mathrm{TiO}_{3}$ film on silicon with the data fit to a Cauchy model for the index of refraction. The uncertainty in the reflectivity data and fit are estimated to be $1 \%$.

dispersion of the complex index with wavelength. Since the film is amorphous and transparent over this wavelength range, a Cauchy model [12] was used for the index of refraction's dispersion function. When the film is crystalline, Sellmeier or other models for the dispersion of the complex index may be appropriate [12]. Thickness maps such as shown in Fig. 3 were obtained for these $\mathrm{BaTiO}_{3}-\mathrm{SrTiO}_{3}$ films.

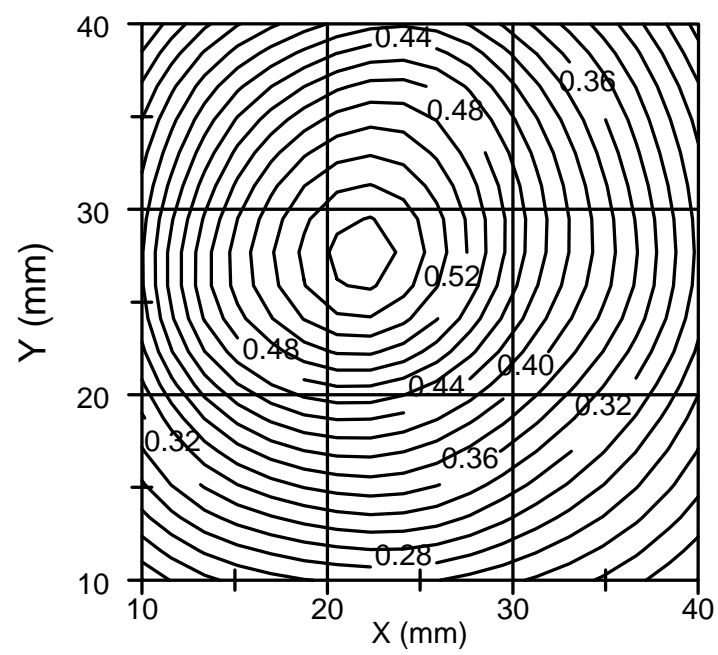

Fig. 3. Thickness map in micrometers from a compositionally graded library film produced by PLD using $\mathrm{BaTiO}_{3}$ and $\mathrm{SrTiO}_{3}$ targets.

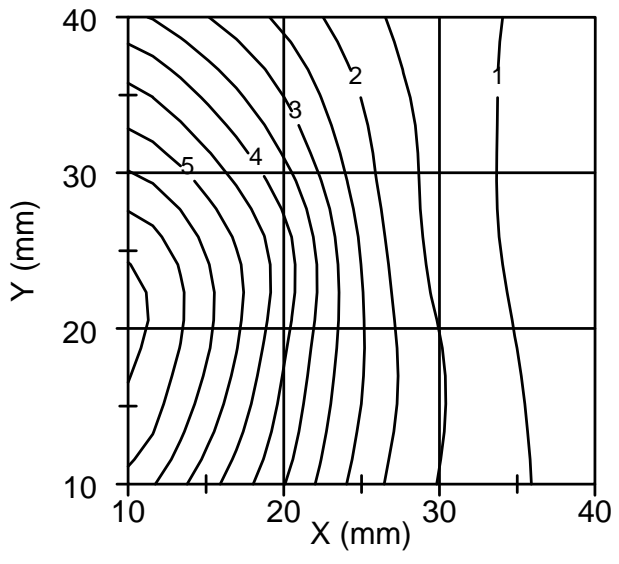

Fig. 4. Map of predicted $\mathrm{BaTiO}_{3} / \mathrm{SrTiO}_{3}$ ratio based on deposition patterns from individual $\mathrm{BaTiO}_{3}$ and $\mathrm{SrTiO}_{3}$ depositions.

\subsection{Characterization of the composition gradient of $\mathrm{BaTiO}_{3}-\mathrm{SrTiO}_{3}$ films}

Thickness maps from single target $\mathrm{BaTiO}_{3}$ and $\mathrm{SrTiO}_{3}$ depositions were produced. When added together, the sum of the single target depositions was essentially identical to the map obtained from the dual target deposition. The composition ratio of the dual target film was predicted by adjusting the individual thickness maps for relative density (assumed to be the same ratio as for bulk) to determine relative atomic fraction. The resulting composition map for the $\mathrm{BaTiO}_{3}-\mathrm{SrTiO}_{3}$ library film is shown in Fig. 4. The experimental conditions of the deposition can be adjusted to produce films with better thickness uniformity and tailored composition gradients [9].

\subsection{Characterization of the reflectance and thickness of n-GaN films}

$n$-GaN films of $5 \mu \mathrm{m}$ nominal thickness deposited on c-sapphire by hydride vapor phase epitaxy (HVPE), were obtained from a commercial source for metallization studies [10]. These films were characterized as received by spectroscopic reflectometry (and transmission) to determine the uniformity of the film thickness. Since the films were several micrometers thick, the spectra contained many oscillations as illustrated in Fig. 5. A simple model is often used in 


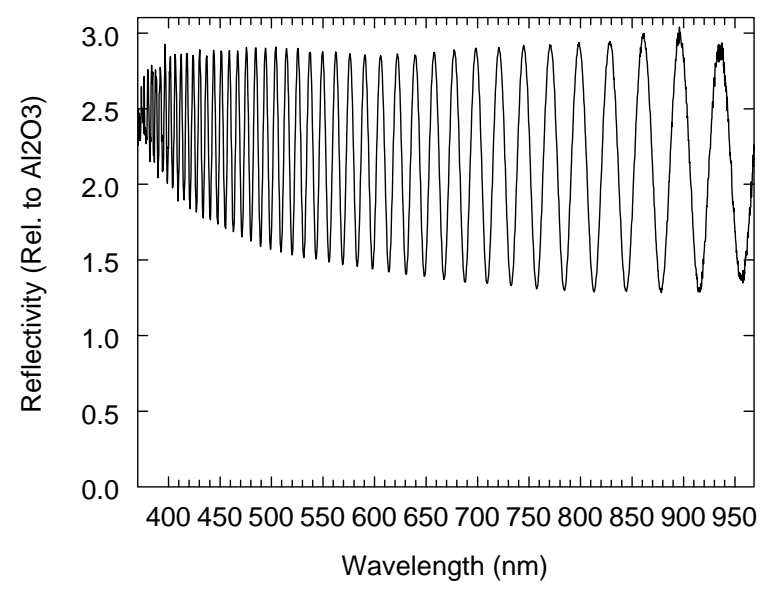

Fig. 5. Reflectance spectrum (relative to sapphire) of a thick GaN film on sapphire.

spectroscopic reflectometry to fit the spectra from a single layer film:

$R=A+B \cos \left(\frac{4 \pi n d}{\lambda}\right)$

where $A$ and $B$ are constants, $n$ the real index of refraction, $d$ the film thickness, and $\lambda$ is the wavelength. It proved difficult to model and fit the thick $n$-GaN film. For this reason Fourier transform spectroscopy was applied to the analysis of the spectra. The raw reflection data were converted from wavelength space to $n / \lambda$ space ( $\propto$ energy) to obtain the frequency proportional to $d$, the film thickness. Since $n$ is dispersive, this was included in the conversion using

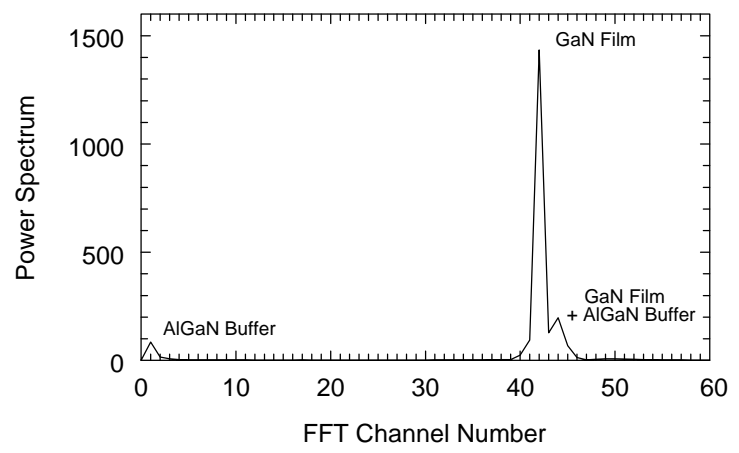

Fig. 6. Fourier transform of thick GaN film reflectance spectrum in $n / \lambda$ space ( $\propto$ energy). Film thickness is determined by multiplying the channel number by $106 \mathrm{~nm}$. values of $n$ for $n$-GaN [13]. The resulting Fourier transform is shown in Fig. 6, where the thickness is proportional to channel number. Evident in the transform are three peaks corresponding to the thin $\mathrm{AlGaN}$ buffer layer $(<300 \mathrm{~nm})$, the $n$-GaN film $(\approx 5 \mu \mathrm{m})$ and the sum of the two layers. The appearance of three peaks is expected in a full analysis of a dual layer film [12]. The $n$-GaN film was found to vary from 4.5 to $6.8 \mu \mathrm{m}$ over the $2 \mathrm{in}$. wafer.

\subsection{Characterization of Au/Ni contacts on GaN}

The combinatorial library film with $\mathrm{Au} / \mathrm{Ni}$ contacts [10] was mapped both in reflectivity and transmission using the spectroscopic reflectometer. Transmission and reflection data were taken from 250 to $1000 \mathrm{~nm}$. In some regions of the library film the transmission spectra were taken relative to a neutral density filter of optical density 2 (method of extension) to complete the map of transmissivity.

Because the metal layers were very reflective the presence of the gallium nitride film only produced small oscillations in the reflection spectrum of the metallized GaN. The results were difficult to model as the $\mathrm{Au}$ and Ni layers have different spectral characteristics and their presence dominated the spectra. Fig. 7 shows the map of the average reflectivity in the $450-500 \mathrm{~nm}$ wavelength range for the metallized library film before and after annealing at $400{ }^{\circ} \mathrm{C}$ for $60 \mathrm{~s}$ in flowing argon. According to XRD, as-deposited and annealed $\mathrm{Au}$ and Ni layers were epitaxial or highly textured with $\left(\begin{array}{lll}1 & 1 & 1\end{array}\right)$ preferred orientation. The nickel rich portion of the library film is much more reflective than the gold rich area in the measured wavelength range. The post-anneal map shows that the reflectivity of Ni/Au bi-layer has increased. This is consistent with improved crystallinity of the metals observed by XRD: intensities of the $\left(\begin{array}{lll}1 & 1 & 1\end{array}\right)$ reflections of both $\mathrm{Au}$ and Ni have increased two to three-fold due to anticipated densification and restructuring of metal layers as a result of the annealing process. The corresponding transmissivity maps are consistent with the reflectivity maps. The observed lower reflectivity (and therefore higher transmissivity) of Au-rich compositions compared to Ni-rich ones at $450-500 \mathrm{~nm}$ wavelengths should be taken into consideration when designing metallization schemes for optically transparent contacts. 

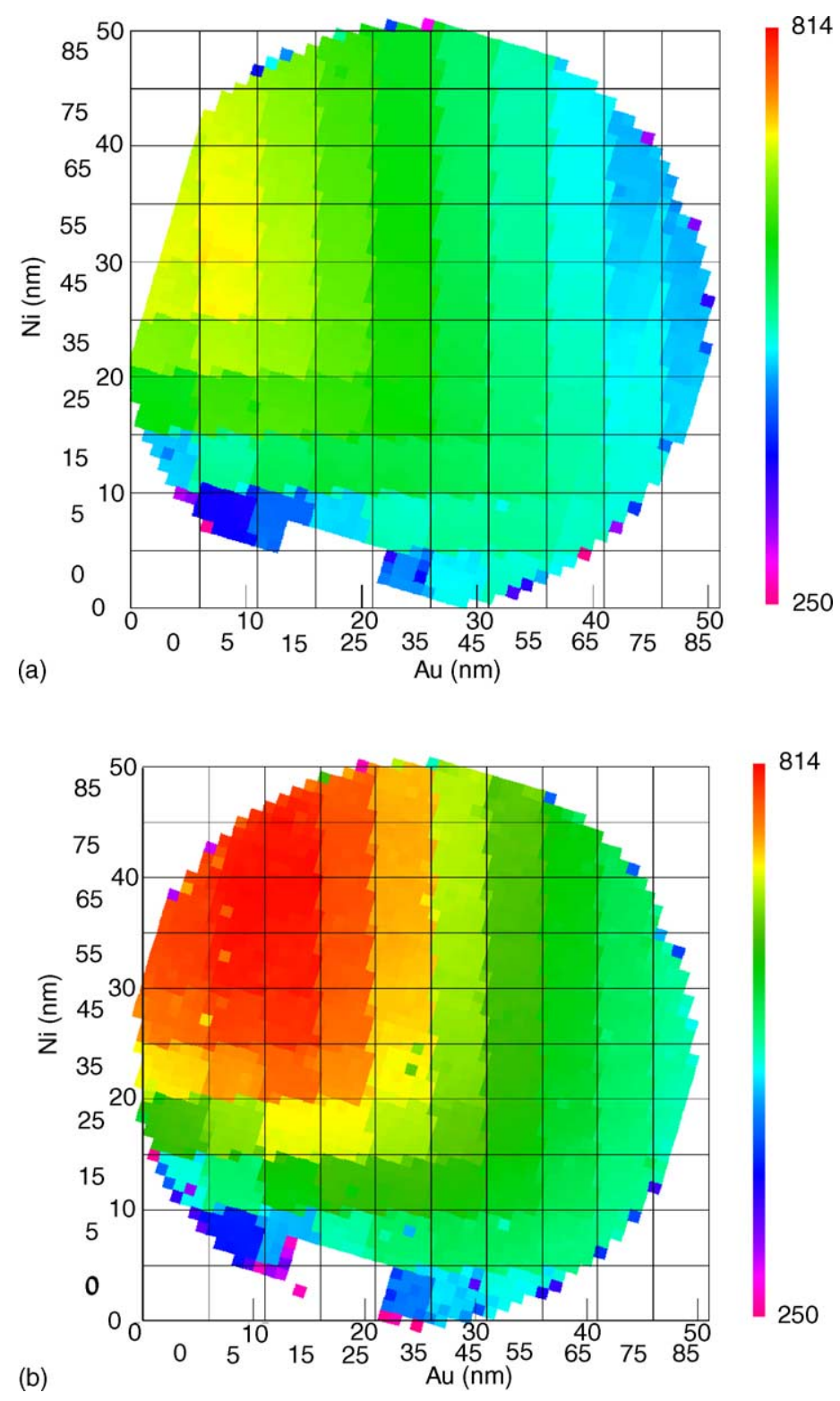

Fig. 7. Reflectivity maps (450-500 nm) of combinatorial Au-Ni contacts on GaN. Both Ni thickness (nm) and position (mm) are indicated on the $Y$-axis. Both Au thickness $(\mathrm{nm})$ and position $(\mathrm{mm})$ are indicated on the $X$-axis: (a) before annealing and (b) after annealing.

\section{Conclusions}

Automated spectroscopic reflectometry is an excellent high throughput characterization tool for measuring film thickness and index of refraction in compositionally varying combinatorial thin films. Thickness maps of thin films produced with single target PLD depositions can be used to predict the thickness and composition of films produced with multiple target PLD depositions. The metallization of wide-band-gap semiconductors can be characterized both in reflectivity and transmission by optical spectroscopy before and after processing. Both differences and changes in the crystallinity of the metal 
contacts can be mapped by these optical techniques and correlate well with XRD measurements.

\section{Acknowledgements}

The authors would like to thank L. Robins (NIST) for $n(\lambda)$ data for GaN, D. Josell (NIST) for e-beam deposition of $\mathrm{Ni}$ and $\mathrm{Au}, \mathrm{V}$. Dmitriev (TDI) for supplying GaN/sapphire samples, and Mr. K.-S. Chang (University of Maryland) for doing the XRD measurements on the $\mathrm{Au} / \mathrm{Ni}$ contacts.

\section{References}

[1] J.J. Hanak, J. Mater. Sci. 5 (1970) 964.

[2] X.D. Xiang, X. Sun, G. Briceno, Y. Lou, K.-A. Wang, H. Chang, W.G. Wallace-Freedman, S.-W. Chen, P.G. Schultz, Science 268 (1995) 1738.
[3] J.F. Scott, Ferroelectrics 183 (1996) 51.

[4] J.F. Scott, Ferroelectr. Rev. 1 (1998) 1.

[5] S. Nakamura, G. Fascol, The Blue Laser Diode, Springer, Heidelberg, 1997.

[6] J.K. Sheu, Y.K. Su, G.C. Chi, P.L. Koh, M.J. Jou, C.M. Chang, C.C. Liu, W.C. Hung, Appl. Phys. Lett. 74 (1999) 2340 .

[7] A. Motayed, A.V. Davydov, L.A. Bendersky, M.C. Wood, M.A. Derenge, D.F. Wang, K.A. Jones, N.S. Mohammad, J. Appl. Phys. 92 (2002) 5218.

[8] D.B. Chrisey, G.K. Hubler, Pulsed Laser Deposition of Thin Films, Wiley/Interscience, New York, 1994.

[9] P.K. Schenck, D.L. Kaiser, Chemistry in Britain 39 (2003) 45.

[10] A.V. Davydov, et al., this issue.

[11] H.G. Tompkins, W.A. McGahan, Spectroscopic Ellipsometry and Reflectometry, A User's Guide, Wiley/Interscience, New York, 1999.

[12] O.S. Heavens, Optical Properties of Thin Solid Films, Dover, New York, 1991.

[13] L.H. Robins, private communication. 